\title{
Un Estudio Descriptivo sobre el Acoso y Violencia Escolar en la Educación Obligatoria
}

\author{
Vicente Félix Mateo ${ }^{1,2}$, Manuel Soriano Ferrer ${ }^{1}$ y Carmen Godoy Mesas ${ }^{2}$ \\ 'Departamento de Psicología Evolutiva y de la Educación. Facultat de Psicologia. Universitat de València.

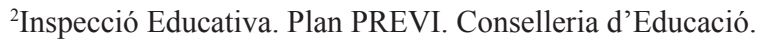

Disponible online 30 de abril de 2009

\begin{abstract}
El presente trabajo analiza la violencia en la población escolar no universitaria de la Comunitat Valenciana, a través de los datos recogidos a través de un Registro On-line. En términos generales, el análisis de los datos señala que las agresiones son más frecuentes en el primer ciclo de Enseñanza Secundaria, donde consecuentemente se abren mayor número de expedientes disciplinarios. Se constata que la violencia más frecuente es la violencia física, seguida de la violencia verbal, seguida con menor incidencia la violencia contra la propiedad, por exclusión y por último, la violencia sexual. Respecto a la topografía, la mayoría de incidencias ocurren en el aula, seguidas del patio, pasillos y alrededores del centro. Los actos violentos no pasan desapercibidos: compañeros, tutor y profesores advierten frecuentemente su existencia, siendo la familia quien reporta los hechos en menor medida. Suelen estar implicados uno o dos participantes, los hombres son más propensos a ser agresores, pero no suelen existir diferencias de género en cuanto a las víctimas, a excepción del primer ciclo de la E.S.O., donde aparecen más víctimas hombres.
\end{abstract}

Palabras clave:

violencia escolar, educación obligatoria, registro on line.

The present study analyses the violence in a non university school population at the Valencian Community, thorough dates obtained from an online register. In general, analysis of data shows that aggression is more often on the first courses of Secondary Education, where more disciplinary measures are used. Physical and verbal violence are more often than violence against property, social exclusion or sexual violence. According to topography, most part of incidences occurs in classrooms, courtyards, corridors and around the school. Acts of violence are not invisible: Pupils, tutors and teachers recognize violent acts, but families report them less often. There are one or two people implicated in acts of violence. Percentage of men is bigger than women as bullies, but there are no sex differences when we talk about victims, except the first courses of secondary school, where there are more male victims.

Keywords:

School violence, obligatory education, online register

Correspondencia: Vicente Félix Mateo (E-mail: Vicente.Felix@uv.es). Manuel Soriano Ferrer (E-mail: Manuel.Soriano@uv.es). Departamento de Psicología Evolutiva y de la Educación. Facultad de Psicología. Universidad de Valencia. Avda. Blasco Ibaññez, 21, 46010 Valencia. Teléfono: (+34) 9639838 49. Fax: (+32) 963864671. 
Durante las últimas décadas, los medios de comunicación han señalado un incremento notorio de la criminalidad, la disruptividad y la violencia en las escuelas. Sin embargo, los datos actuales no dan apoyo empírico a la idea de que en los últimos años se haya producido un dramático incremento de la violencia escolar (Burns, Dean y Jacob-Timm, 2001; Furlong y Morrison, 1996). De hecho, los estudios comparativos ponen de manifiesto que las escuelas constituyen uno de los lugares más seguros para los niños y los jóvenes en edad escolar. Robos, asaltos y crímenes son más probables en los domicilios particulares o en la calle antes que en los colegios, así como la casuística de abusos sexuales, donde el 91\% de los mismos son perpetrados por miembros de la propia familia y en el entorno más cercano (Hyman y Perone, 1998).

No obstante, los problemas relacionales existentes en los centros constituyen muchas veces el reflejo de lo que ocurre fuera de ellos (Marín, 2002; Rodríguez, Gutiérrez, Herrero, Cuesta, Hernández et al., 2002). Así, aunque las escuelas constituyan en general lugares seguros, también se producen a veces problemas de convivencia que hacen referencia a conducta antisocial -robos, vandalismo; daños hacia la propiedad, agresividad -violencia física y verbal hacia los profesores o resto de alumnos (Astor, Pitner, Benbenishty y Meyer, 2002) acoso escolar o violencia sexual (Félix, Godoy y Martínez, 2008) que deben tener una respuesta rápida e inmediata por parte de los centros, la administración educativa, y la sociedad en general.

El problema de la violencia escolar es multifacético, ya que "implica actos criminales y agresiones en las escuelas que inhiben el desarrollo del aprendizaje y daña el clima escolar" (Furlong y Morrison, 2000; p. 17). Los participantes en los actos de violencia asumen diferentes roles: el acosador, el seguidor de éste, el que refuerza la situación, el que opta por escapar cuando se acosa a otra persona, así como la víctima (Salmivalli, Lagerspetz, Björkqvist, Österman y Kaukiainen, 1996). La violencia escolar abarca violencia física (golpes, puñetazos, patadas), violencia verbal (amenazas, burlas, motes, rumores maliciosos, etc.) así como la exclusión social, en la que una persona es deliberadamente ignorada con la finalidad de hacerle daño (Wolke, Woods, Bloomfield y Karstadt, 2000), y violencia sexual (tocamientos no consentidos, relaciones sexuales forzadas, etc.) (Lacasse, Purdy y Mendelson, 2003).

El acoso escolar (del inglés bullying) se ha convertido en un tópico internacional. Aunque no existe todavía un consenso en la comunidad científica sobre su conceptualización, muchos investigadores coinciden en señalar que se trata de un acto con la finalidad de provocar daño, que se realiza de forma sistemática y repetida, y con desigualdad de poder entre el agresor y la víctima (Farrington, 1993), donde se practica un abuso sistemático de poder (Rigby 2002). Otros aspectos aplicables a la definición harían referencia a que la persona abusada se siente coaccionada, degradada, humillada, amenazada, intimidada o asustada (Monks, Smith, Naylor, Barter, Ireland et al., 2009). Investigaciones recientes han identificado nuevas formas de acoso, como el cyberbullying, entendido como el daño repetido e intencionado ocasionado a través del medio electrónico (Patchin e Hinduja, 2006). Por último, aunque no por ello menos grave, existe otra forma de acoso que haría referencia al abuso sexual. Abarcaría actos no consentidos como caricias inadecuadas, contacto sexual, etc. También deben incluirse otros actos como: exposición indecente; masturbación exhibicionista; exposición directa a la pornografía; explotación sexual; menosprecios o vejaciones sexuales, etc. Aunque en algunos casos pueden ser "relaciones consentidas" (Canavan, Meyer y Higgs, 1992), habitualmente se produce bajo condiciones de desigualdad de poder, amenazas y fuerza, antes, durante o después de los actos de abuso del violador frente a la víctima.

En el contexto escolar, la mayoría de las agresiones se producen en el patio de recreo, el aula y los pasillos. Los autoinformes de los alumnos revelan que la edad de padecer un acoso escolar oscila entre el período de los 8 a los 16 años. En cuanto al género, los hombres son más proclives a ser agresores, pero no existen diferencias de género en cuanto a las víctimas. En cuanto a las características de las agresiones, los hombres utilizan/reciben más las agresiones físicas, mientras que las mujeres utilizan más la forma indirecta y relacional del acoso (Olweus, 1993).

La prevalencia de la violencia escolar varía enormemente en función de diferentes factores como el método de recogida de la información (p.e. registro directo desde el centro, autoinformes retrospectivos de los alumnos, etc.), la definición que se utiliza de violencia (p.e si incluye acoso directo e indirecto), el tamaño de la escuela o del aula, contexto escolar rural vs. urbano, etc. De hecho, aunque factores tales como tamaño del colegio (las escuelas grandes son más proclives a la violencia), la pobreza o el nivel de criminalidad de la zona donde está ubicado el centro, la etapa educativa de los alumnos (los estudiantes de secundaria tienen mayor probabilidad de padecer reyertas, peleas, etc.) constituyen buenos predictores de la violencia escolar, son las actitudes del profesorado ante las situaciones de acoso, el grado de supervisión de las actividades en el tiempo libre y la existencia de una política escolar efectiva contra el acoso las que parecen tener mayor significatividad (Nickerson y Martens, 2008). No obstante, los datos de prevalencia sobre la violencia señalan que entre un 5\%-20\% de los alumnos de primaria y secundaria se reconocen como víctimas de algún acto de violencia y, entre el $4 \%-10 \%$ como agresores, aunque algunos alumnos son tanto víctimas como acosadores, y otros agresores/víctimas (Olweus, 1993; Smith, Morita, Junger-Tas, Olweus, Catalano et al., 1999). En concreto, en el estudio realizado por el Defensor del Pueblo (2000), un 33\% se reconoce como víctima de insultos, un $4.1 \%$ de agresiones físicas y un $0.7 \%$ de amenazas con armas. Respecto a los agresores, un $40.9 \%$ reconoce haber insultado, un $6.6 \%$ han agredido físicamente y un $0.3 \%$ han amenazado con armas.

Partiendo de estas consideraciones previas, en el presente estudio se pretende analizar algunas de las características de 
la violencia escolar en las etapas de escolaridad obligatoria en Valencia y su provincia.

\section{Método}

\section{Participantes}

En este estudio participaron 282 centros de enseñanza no universitaria (públicos y concertados) de la Comunitat Valenciana, concretamente de la ciudad de Valencia y su Provincia, en los que se produjo algún incidente relacionado con problemas en la convivencia escolar, y que lo hubiesen registrado on line a través del Registro Central de Incidencias (instrumento de evaluación). Los datos recogidos en este trabajo hacen referencia al periodo comprendido desde septiembre de 2007 hasta diciembre de 2008. En particular, analizamos 1083 incidencias registradas en el Registro Central del Plan PREVI. Estas incidencias provienen de todas las etapas educativas obligatorias, registradas por los centros en función de la gravedad que ellos mismos establecieron de los hechos.

\section{Instrumentos}

El Registro Central (ver Figura 1) es un instrumento de recogida de información on line sobre las incidencias que, de forma significativa, alteran la convivencia escolar en los centros educativos no universitarios de la Comunitat Valenciana. Según el Decreto 39/2008, de 4 de abril, del Consell, (DOCV 5752) sobre la convivencia en los centros docentes no universitarios sostenidos con fondos públicos, todos los centros tienen la obligación de registrar aquellas incidencias que impliquen algún tipo de violencia, que se produzcan tanto dentro como fuera del centro educativo, y en la que estén implicados de forma directa o indirecta alumnos/as del centro.

Técnicamente, presenta una estructura de formulario electrónico, siendo la mayoría de campos de activación a través del cursor, verificando cada una de las casillas que lo componen, a excepción de algunos campos de tipo descriptivo donde se puede escribir directamente. La introducción de datos es dependiente de la previa introducción de una contraseña proporcionada a cada centro educativo, de la cual sólo tienen acceso los directores/as e inspectores/as de los mismos, siendo así toda la información que se transmite anónima (no aparecen datos de filiación de los alumnos ni alumnas implicados), y privada (no es de acceso público). Este instrumento on line permite un acercamiento directo a la realidad de los centros, siendo un medidor más fiable, objetivo y con mayor validez ecológica de la convivencia, que la información aportada por autoinformes, que suele ser mucho más variable debido a las diferentes formas de recoger la información (Sawyer, Bradshaw y O'Brennan, 2008).

\section{Procedimiento}

El acceso al Registro Central de Incidencias se realiza a través de la página WEB de la Conselleria d'Educació (http://appweb2.cult.gva.es/hdfi41/login.jsp), introduciendo una identificación (el código de centro) y una clave (cada centro dispone de la suya). Una vez que el centro ha cumplimentado los datos, aparece otra pantalla accediendo al formulario electrónico (ver Figura 1), que incluye los campos para definir específicamente el tipo de incidencia que se ha producido. Necesariamente se deberán cumplimentar "Fecha de comisión", "Edad de la víctima" y "sexo", además de relativas a la incidencia. Finalmente, se debe pulsar al botón "Alta" para registrar definitivamente la incidencia. Al final del formulario, el inspector/a del centro, cumplimenta una serie de campos relacionados con las funciones de la Inspección Educativa, una vez que es conocedor de la incidencia (cuando el centro registra la incidencia, le aparece al inspector/a de forma automática en su correo electrónico).

Figura 1. Registro Central de Incidencias (online).

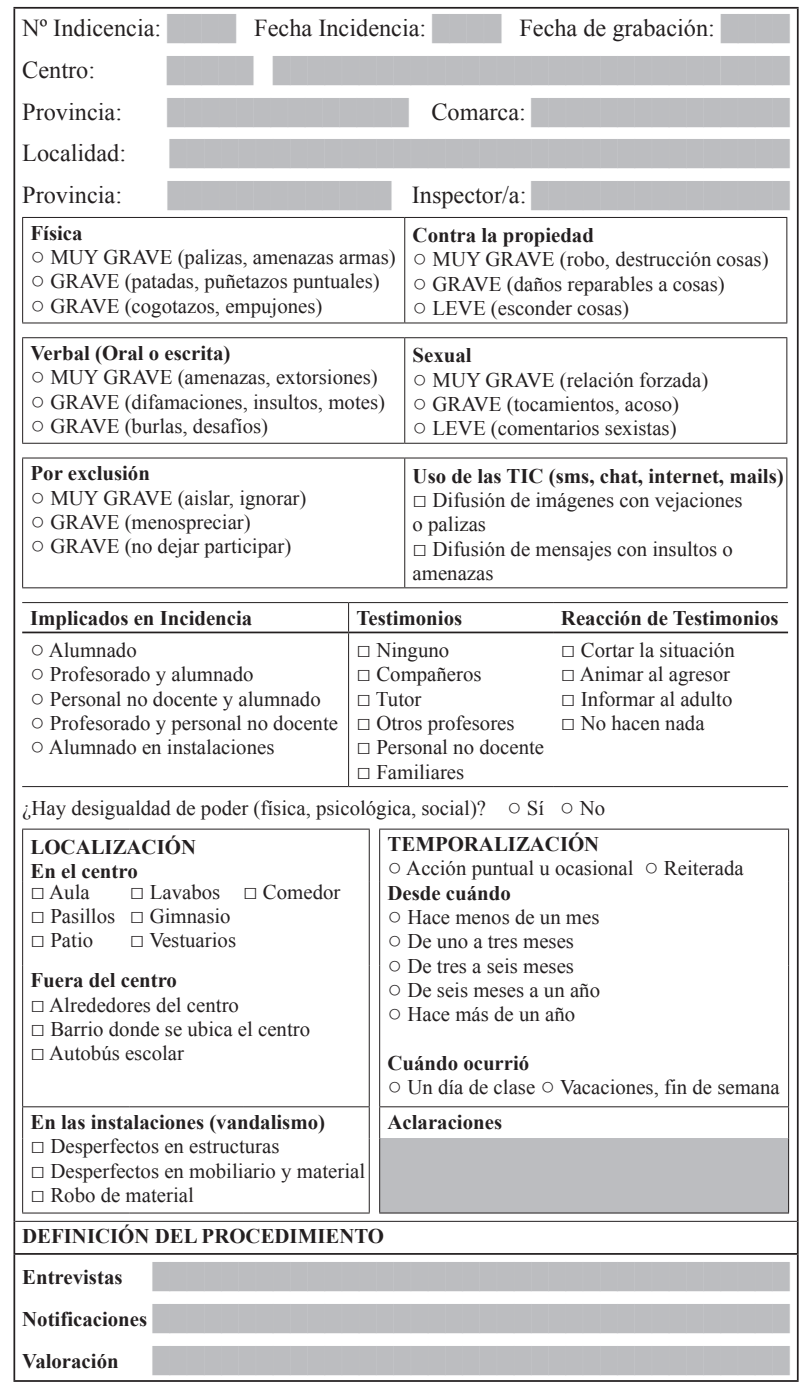


Resultados

Desde septiembre de 2007 a diciembre de 2008 se han registrado un total de 1083 incidencias. Respecto a ese total analizaremos a continuación los datos referentes a la proporción de centros públicos y concertados que han registrado algún tipo de incidencia relacionada con la convivencia, los tipos de violencia utilizados más frecuentemente y su gravedad (estimada por los propios centros educativos), así como qué porcentaje de incidentes relacionados con la convivencia escolar llevan asociados un parte disciplinario. Analizaremos cual es la topografía de los actos violentos en las escuelas y alrededores, quienes suelen ser los testigos de las agresiones, cual suele ser su rol ante una agresión, cuántos agresores suelen participar en los actos violentos y la utilización de las nuevas tecnologías en los actos de violencia.

Registro de incidencias por titularidad. El primer dato que analizaremos de los proporcionados por el Registro Central hace referencia a la titularidad del centro (público y concertado). En este sentido, observamos (ver Tabla 1) que aproximadamente una cuarta parte de las incidencias registradas (24\%) pertenecen a centros concertados. El 76\% restante de las incidencias pertenecen a centros públicos.

Tabla 1. Número y porcentaje de incidencias por titularidad.

\begin{tabular}{lcc}
\hline Titularidad & $\mathrm{N}^{\circ}$ incidencias & Porcentaje \\
\hline Público & 823 & 76 \\
Concertado & 260 & 24 \\
Total & 1083 & 100,0 \\
\hline
\end{tabular}

Registro de incidencias según tipo de violencia ejercida. En este apartado se describe la distribución del número de incidencias por tipo de violencia, mostrando los porcentajes que representan respecto del total de incidencias registradas en el período analizado. En cuanto a la tipología de las incidencias que se producen en el contexto escolar, destacan (ver Tabla 2) las incidencias por violencia física (42\%) y violencia verbal (40\%) sobre el resto que se manifiestan en porcentajes mucho menores.

Tabla 2. Frecuencia y porcentaje de los tipos de violencia detectados por severidad

\begin{tabular}{lcccccccc}
\hline & \multicolumn{1}{c}{ Muy Grave } & \multicolumn{2}{c}{ Grave } & \multicolumn{2}{c}{ Leve } & \multicolumn{2}{c}{ TOTAL } \\
\hline & $\mathrm{N}$ & $\%$ & $\mathrm{~N}$ & $\%$ & $\mathrm{~N}$ & $\%$ & $\mathrm{~N}$ & $\%$ \\
Física & 153 & 9.1 & 414 & 24.7 & 129 & 7.7 & 696 & 42 \\
Propiedad & 61 & 3.6 & 63 & 3.8 & 13 & 0.8 & 137 & 8 \\
Verbal & 275 & 16.4 & 301 & 17.9 & 95 & 5.7 & 671 & 40 \\
Sexual & 6 & .3 & 33 & 2.0 & 15 & .9 & 54 & 3 \\
Exclusión & 13 & 0.8 & 89 & 5.3 & 16 & 1.0 & 118 & 7 \\
TOTAL & 508 & 30.2 & 900 & 53.7 & 268 & 16.1 & 1676 & 100 \\
\hline
\end{tabular}

Tal y como aparece reflejado en la Tabla 2, la violencia física y verbal representan el $82 \%$ del total de incidencias registradas, mientras que el resto de tipos de violencia se sitúan a niveles significativamente inferiores (contra la propiedad el 8\%; violencia por exclusión el 7\%; y violencia sexual en torno al 3\%).
Dentro de cada tipología mostramos las distribuciones según la gravedad del tipo de incidencia: leve, grave y muy grave respecto del total de incidencias registradas en el período. La valoración de la gravedad la establecen los centros al registrar las incidencias, basándose en su propio Reglamento de Régimen Interno, así como en el Decreto 39/2008 de Convivencia en los centros docentes. De forma general, podemos concluir que las faltas graves representan el $53.7 \%$ del total de las faltas, seguidas de las muy graves, con un $30.2 \%$. Observamos igualmente en la Tabla 2 que el mayor porcentaje de incidencias catalogadas como muy graves provienen de la violencia verbal; mientras que dentro de las graves y leves destaca la violencia física. Respecto al resto de tipos de violencia (sexual y por exclusión), son catalogadas como graves, aunque su incidencia es significativamente menor que las otras.

Expedientes disciplinarios a agresores/as. El Decreto 39/2008 de 4 abril, sobre la convivencia en los centros docentes no universitarios sostenidos con fondos públicos (Núm. 5738) establece que los centros educativos, a través de su reglamento de régimen interior, incluirán el conjunto de normas por los que se regule y favorezca la convivencia, fomentando el adecuado clima escolar, agilizando en caso de conflicto, la aplicación de medidas correctoras, garantizando así los derechos y deberes de la comunidad educativa.

Como puede observarse en la Gráfica 1, el 58.2\% de los expedientes disciplinarios se aglutinan en el primer ciclo de la educación secundaria obligatoria, reduciéndose a poco más del $18.3 \%$ en el segundo ciclo de esta etapa. Respecto a la educación primaria, es en el último ciclo donde se produce un mayor porcentaje (14.6\%). Resaltar que en nuestros datos aparece un solo caso de aplicación de expediente disciplinario en la educación infantil.

Gráfica 1. Expedientes disciplinarios por etapa educativa.

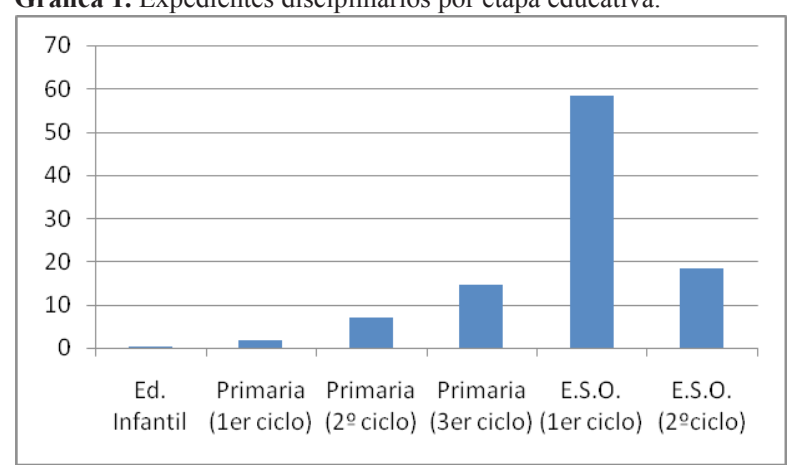

Localización espacial de la agresión. En cuanto a la topografía de las incidencias dentro del centro se dan con más frecuencia, y por este orden, en el aula (30.9\%), patio (24.4\%), pasillos $(15.1 \%)$ y alrededores del centro $(10.8 \%)$. El resto de escenarios (lavabos, gimnasio y vestuarios) tienen una incidencia apenas significativa respecto a las anteriores localizaciones (ver Gráfica 2). 
Gráfica 2. Topografía de los actos violentos.

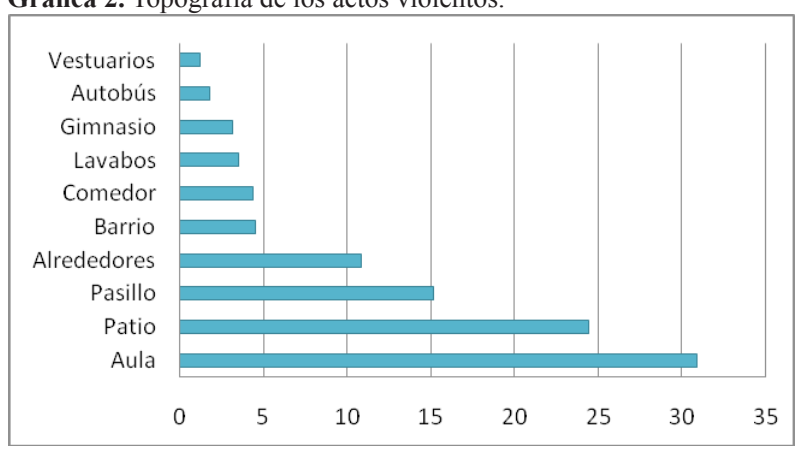

Testigos de las agresiones. Los testigos más frecuentes (ver Tabla 3) son los compañeros $(72.8 \%)$ y los docentes $(63.6 \%)$. Otro aspecto relevante es que compañeros, tutores y profesores son los primeros en ser conocedores e informar de los conflictos y situaciones de violencia, mientras que las familias reportan estos hechos en un porcentaje significativamente menor.

Tabla 3. Informantes de los actos violentos en el contexto escolar.

\begin{tabular}{lcc}
\hline Informantes & $\mathrm{N}$ & $\%$ \\
\hline Compañeros & 789 & 72.8 \\
Tutor & 249 & 23.0 \\
Profesores & 429 & 39.6 \\
Personal no docente & 164 & 15.1 \\
Familia & 91 & 8.40 \\
\hline
\end{tabular}

Implicados en los actos violentos. En cuanto al número de personas implicadas en las incidencias registradas, podemos observar en la Tabla 4, que el 55.49\% de las incidencias registradas, implican a un agresor, mientras que en el $30.94 \%$ participaron dos personas. El porcentaje de incidencias con más de 3 personas alcanza porcentajes inferiores al 3\%.

Tabla 4. Número de alumnos implicados.

\begin{tabular}{lcc}
\hline Implicados & $\mathrm{N}$ & $\%$ \\
\hline 1 implicado & 601 & 55.49 \\
2 implicados & 335 & 30.94 \\
3 implicados & 42 & 3.88 \\
4 implicados & 27 & 2.49 \\
5 implicados & 15 & 1.39 \\
NS/NC & 63 & 5.81 \\
\hline
\end{tabular}

Papel de los iguales en las conductas violentas. Respecto a la reacción de los testigos ante una situación de violencia, el comportamiento habitual ha sido: cortar la situación (en un 42.4\%), animar al agresor (4.43\%), informar a un adulto $(29.28 \%)$ o no hacer nada (17.45\%). En el resto de incidencias $(6 \%)$ esta variable no fue consignada por los centros educativos objeto de estudio.
Victimas y agresores por género y etapa educativa. Como aparece reflejado en la Gráfica 3, es en el primer ciclo de la E.S.O. donde más problemas relacionados con la convivencia se producen de toda la etapa educativa obligatoria, explicando aproximadamente el $45 \%$ del total de incidencias que se producen entre los 3 y los 16 años. Por otra parte, respecto a las víctimas y agresores, podemos concluir que existe cierta igualdad en el porcentaje a lo largo de las distintas etapas educativas, es decir, el número de agresores y de víctimas suele ser similar.

Gráfica 3. Víctimas y agresores por etapa educativa.

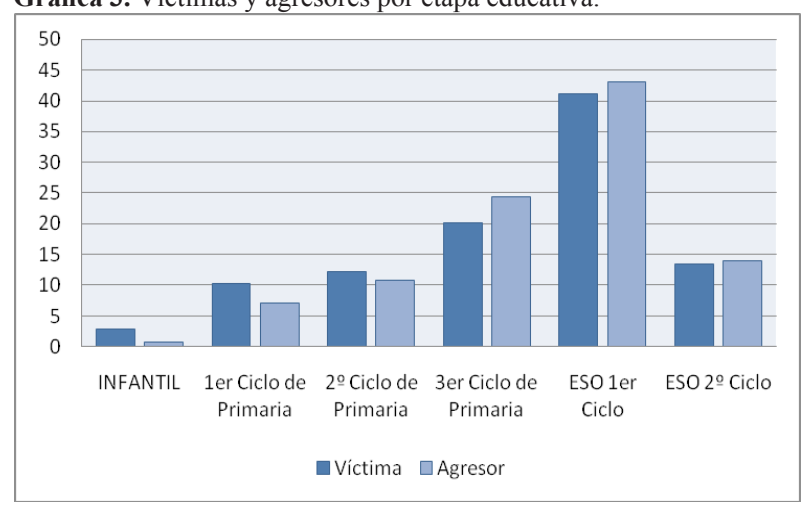

Respecto al género del agresor, tal y como aparece reflejado en la Gráfica 4, podemos observar que los hombres adoptan un rol agresor de forma mayoritaria a lo largo de la etapa educativa obligatoria, representando un $67 \%$, mientras que las mujeres agresoras representan un $33 \%$ del total.

Por último, respecto al rol de las víctimas, podemos observar en la Gráfica 5 que el porcentaje de hombres y mujeres víctimas suele ser similar en la mayoría de etapas educativas, siendo siempre superior, aunque de forma discreta, el número de hombres. Una excepción constituye el primer ciclo de la E.S.O. donde los hombres son más propensos a ser victimizados que las mujeres.

Gráfica 4. Agresores por género.

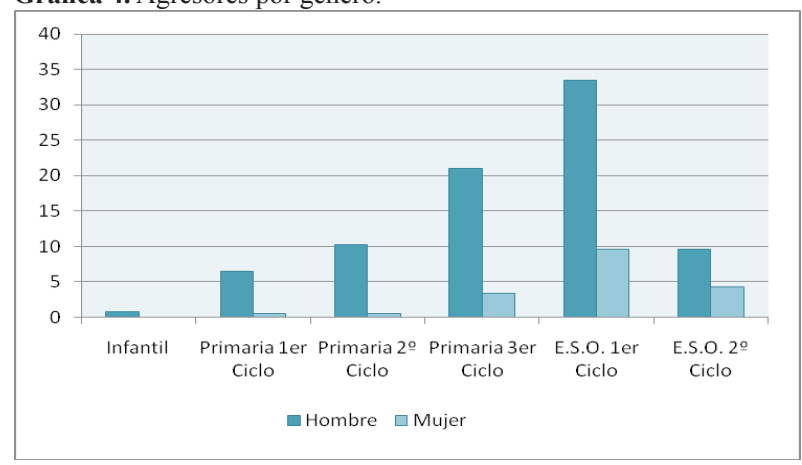


Gráfica 5. Víctimas por género.

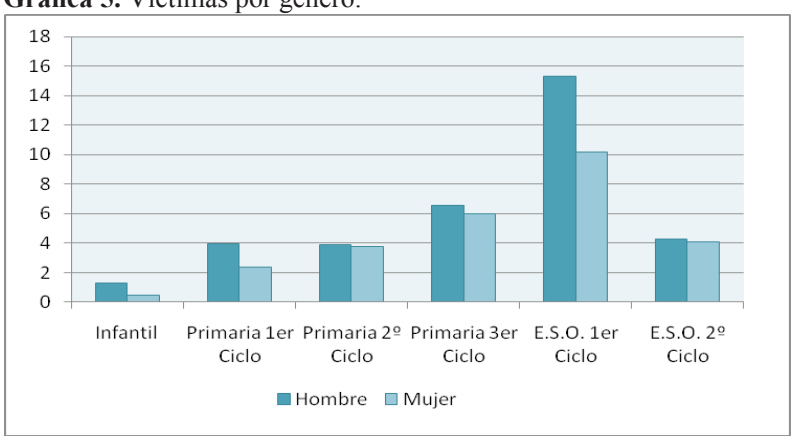

Un último dato respecto a la violencia haría referencia a las agresiones de alumnos a docentes. En este sentido, nuestros datos muestran que suponen un total del $10 \%$ del total de incidencias. En cuanto al género, 31 incidencias (28.2\%) tuvieron como víctima a un profesor, mientras que las 79 restantes $(71.8 \%)$ fueron dirigidas hacia una profesora.

Violencia relacionada con el uso de nuevas tecnologías. Respecto a las incidencias que implican la utilización de algún medio relacionado con las nuevas tecnologías, el Registro Central divide entre las conductas de violencia con difusión de imágenes y mediante mensajería móvil (sms). En el primer caso, (difusión de imágenes), se han dado 16 incidencias a lo largo del periodo analizado, representando el $1.4 \%$ del total de incidencias. En el segundo caso, (acoso mediante sms), se da en 29 incidencias, representando el $2.6 \%$ del total de incidencias. Así pues, en el 4\% de las 1086 incidencias registradas se utilizaron las nuevas tecnologías.

Incidencias con posibles casos de acoso escolar. El Registro Central de Incidencias contempla el acoso o bullying cuando una incidencia se ha ejercido sobre una o más víctimas, activando la casilla de desigualdad física o psicológica, siendo invisible a los adultos y mostrando reiteración en el tiempo. Durante los meses de septiembre de 2007 hasta diciembre de 2008 se registraron 10 incidencias de posible acoso escolar, suponiendo una prevalencia del $0.9 \%$ del total de las incidencias registradas.

\section{Discusión}

Este estudio se propuso realizar un análisis de la violencia escolar en los centros públicos y concertados de Valencia y su provincia, a partir del análisis de los datos consignados on line por los propios centros en el Registro Central de Incidencias.

En primer lugar, respecto a la titularidad de los centros, observamos que los centros públicos han registrado tres veces más incidencias que los centros concertados. Esta distribución no se corresponde exactamente con la distribución existente por titularidad de los centros en la Comunitat Valenciana (62.4\% públicos y $37.6 \%$ concertados) y tampoco por el número de alumnos matriculados en ellos $(68.5 \%$ públicos, $27.74 \%$ concertados; el 3.6\% restante hace referencia a alumnos matriculados en centros privados). Mediante el uso de autoinformes cumplimentados por los propios alumnos, el Defensor del Pueblo (2000) no observa diferencias significativas entre las escuelas públicas y privadas.

En segundo lugar, al igual que en el estudio del Defensor del Pueblo (2000), son la violencia física y la verbal las que se producen con mayor frecuencia, y a su vez las que son catalogadas mayoritariamente como graves o muy graves. El resto de incidencias tienen una prevalencia significativamente menor, distribuyéndose como graves o leves. Cabe destacar que la violencia física o verbal no se produce exclusivamente en el contexto escolar. Pagani, Tremblay, Nagin, Zoccolillo, Vitaro y McDuff (2009) muestran que los adolescentes utilizan típicamente estos tipos de violencia también en el contexto familiar contra sus padres. Por último, la violencia por exclusión representa el 7\%. Pensamos que al igual que en el estudio llevado a cabo por Yoon (2004), algunos profesores piensan que las agresiones físicas o verbales generan más problemas de victimización que la exclusión social, por lo que parece que son más proclives a identificar los otros tipos de violencia.

Tercero, la aplicación de los protocolos de convivencia hace que muchas incidencias catalogadas como graves o muy graves lleven asociado la apertura de un expediente disciplinario. En general, de forma similar al estudio de Muñoz, Pérez y Martín (2006), más de la mitad de los expedientes disciplinarios son tramitados en el primer ciclo de la educación secundaria obligatoria (12-14 años).

Contrariamente a lo que suele creerse, la mayoría de las incidencias ocurren en el aula, el patio, los pasillos y los alrededores del centro. De hecho, aglutinan el $80 \%$ de los actos violentos graves o muy graves de nuestros centros escolares. Resultados similares se han encontrado en otros estudios (DP, 2000; Olweus, 1993, 1994). El estudio del Defensor del Pueblo (2000) señala el patio de recreo como el escenario más frecuente para las agresiones físicas, mientras que los insultos, motes y agresiones contra la propiedad se producen con mayor frecuencia en el aula.

Un aspecto relevante de la localización es que la mayoría de actos contra la convivencia se produzcan en el aula, normalmente en el cambio de un profesor a otro, pero muchas otras con el docente dentro. Así pues, aunque resulta paradójico, la figura de autoridad del profesorado no supone muchas veces un obstáculo para impedir determinados comportamientos disruptivos.

Respecto a los informantes, los datos muestran que son los compañeros y el profesorado quien detecta con mayor frecuencia las situaciones de violencia o acoso escolar. Este hecho demuestra que la invisibilidad no es una característica que domine especialmente en las incidencias registradas. 
En cuanto a la relación entre edad y violencia, nuestros datos han arrojado datos fuera de dudas: las edades comprendidas entre los 12 y los 14 años (primer ciclo de la educación secundaria obligatoria), alcanzan prácticamente el $45 \%$ de todas las incidencias que se producen a lo largo de toda la escolaridad obligatoria (de los 6 a los 16 años), mostrando que en la adolescencia temprana existe un superior riesgo de violencia que en edades posteriores, así como de conductas de riesgo. Consecuentemente, comentamos anteriormente que también en esta etapa educativa es donde más expedientes disciplinarios fueron llevados a cabo por incidencias que alteran gravemente la convivencia en los centros. Estos resultados han sido igualmente encontrados en otros estudios llevados a cabo en el conjunto de la población (Díaz-Aguado, 2005; Díaz-Aguado, Martínez Arias y Martín Seoane, 2004; Eslea y Rees, 2001; Muñoz, Pérez y Martín, 2006)

Respecto al papel de los iguales ante las situaciones violentas, un $42.4 \%$ de los alumnos cortan la situación, resultado similar al de otras investigaciones (Díaz-Aguado, Martínez y Martín, 2004). Pese a que no son muchos los alumnos que optan por animar, pensamos que el número de alumnos que no hacen nada ante una situación de violencia (17.45\%) debería ser inferior, especialmente porque supone una incapacidad para ponerse en el lugar de los demás, dejar que el miedo domine las propias acciones, y en definitiva, ayuda a perpetuar determinados comportamientos ante los que deberíamos tener tolerancia cero.

Al analizar posibles diferencias de género en cuanto al rol de víctima y de agresor, los resultados son contundentes al señalar el papel predominante de los hombres frente a las mujeres como agresores, manifestando un riesgo superior de violencia e intolerancia que las chicas, mientras que no existen apenas diferencias entre las víctimas, siendo similar el porcentaje de hombres y mujeres que han sido victimizados (a excepción de los docentes, donde las profesoras fueron la diana de las agresiones en un $71 \%$ ). Estudios previos en otras poblaciones han encontrado resultados similares, como en la sociedad finlandesa (Sourander y Helstelä, 2000), israelí (Attar y Khoury, 2008), norteamericana (Farrington, 1993), inglesa (Monks et al., 2009), española (Díaz-Aguado, Martínez y Martín, 2004; Félix, Godoy y Martínez, 2008) y la sueca (Olweus, 1991, 1993, 1994). Aunque otros estudios han encontrado mayores índices de victimización en alumnos de 8 años que de 11 años (Borg, 1999; Nansel et al., 2001; Owens et al., 2005; Scheithauer, Hayer, Peterman y Jugert, 2006).

Investigaciones recientes (Beran y Li, 2005; Hinduja y Patchin, 2008) han puesto de manifiesto que aproximadamente del 20-35\% de los alumnos se consideran víctimas de algún tipo de cyberacoso mediante chats, mensajes instantáneos o correo electrónico. En nuestro estudio hallamos una incidencia relativamente baja ( $4 \%$ del total de agresiones), aunque puede convertirse en un medio óptimo para amenazar a otras personas (blogs, páginas web, grabaciones de vídeo a través del móvil,
Messenger, correo electrónico, etc.), con el agravante de la rapidez con que se transmiten y el hecho de que muchos adolescentes piensan que los comentarios que vierten sobre páginas WEB, blogs personales, etc., son anónimos y no tienen ninguna repercusión legal.

A modo de conclusión, una vez analizados los datos, queda patente la necesidad de intervenir ante los problemas de indisciplina y violencia que se producen en los centros. El Plan PREVI abarca diferentes medidas para prevenir la violencia en la escuela, así como para incrementar y promover respuestas prosociales hacia los conflictos (ver revisión en Félix, Soriano, Godoy y Martínez, 2008). Incluye medidas de prevención hacia el Sistema Educativo, con iniciativas para incrementar la supervisión del adulto, y orientaciones para todos los miembros. Medidas de prevención dirigidas a la población en riesgo, con procedimientos para dar respuesta a situaciones de forma urgente y medidas de prevención para la sociedad en general, que fomentan la participación en cuestiones relacionadas con la violencia escolar. El Plan PREVI supone un intento para modificar el clima escolar promocionando un claro mensaje de que la violencia es inaceptable y el reconocimiento de que todos los miembros tienen una responsabilidad directa de actuar y fomentar la participación de la comunidad escolar ante los problemas de convivencia.

\section{Referencias}

Astor, R., Pitner, R.O., Benbenishty, R. y Meyer, H.A. (2002). Public concern and focus on school violence. En L.A. Rapp-Paglicci, A.R. Roberts y J.S. Wodarski (Eds.), Handbook of violence. New York: John Wiley y Sons. Inc.

Attar, S. y Khoury, M. (2008). Indirect versus verbal forms of victimization at school: The contribution of student, family and school variables. Social Work Research, 32, 159-170.

Beran, T. y Li, Q. (2005). Cyber-harassment: A study of a new method for an old behavior. Journal of Educational Computing Research, 32(3), 265-277.

Borg, M.K. (1999). The extent and nature of bullying among primary and secondary school children. Educational Research, 41, 137-153.

Burns, M. K., Dean, V. J. y Jacob-Timm, S. (2001). Assessment of violence potential among school children: Beyond profiling. Psychology in the Schools, 38, 239-247.

Canavan, M. C., Meyer, W. J. y Higgs, D. C. (1992). The female experience of sibling incest. Journal of Marital and Family Therapy, 18, 129-142.

Defensor del Pueblo (2000). Informe sobre violencia escolar: el maltrato entre iguales en la Educación Secundaria Obligatoria. Madrid: Publicaciones de la Oficina del Defensor del Pueblo.

Diamanduros, T., Downs, E. y Jenkins, S. (2008). The role of 
school psychologists in the assessment, prevention, and intervention of cyberbullying. Psychology in the Schools, 45 (8), 693-704.

Díaz-Aguado, M. J., Martínez Arias, R. y Martín Seoane, G. (2004). Prevención de la violencia y lucha contra la exclusión desde la adolescencia. Volumen uno. La violencia entre iguales en la escuela y en el ocio. Estudios comparativos e instrumentos de evaluación. Madrid: Instituto de la Juventud. Disponible también en: mtas.es/injuve/novedades/prevenciónviolencia.htm.

Díaz-Aguado, M.J. (2005). La violencia entre iguales en la adolescencia y su prevención desde la escuela. Psicothema, 17, 549-558

Eslea, M. y Rees, J. (2001). At what age are children more likely to be bullied at school? Aggressive Behavior, 27, 419-429.

Farrington, D. (1993). Understanding and preventing bullying. En M. Tonry (Ed.), Crime and justice: A review of research, 17 (pp. 381-458). Chicago: University of Chicago Press.

Félix, V., Godoy, C. y Martínez, I. (2008). Violencia entre iguales: Resultados de un estudio descriptivo de la provincia de Valencia. Informació Psicològica, 94, 36-48.

Félix, V., Soriano, M., Godoy, C. y Martínez, I. (2008). Prevención de la violencia y promoción de la convivencia escolar en la Comunitat Valenciana (Plan PREVI). Aula Abierta, 36, 97-110.

Furlong, M. y Morrison, G. (1996). Status update of research related to national goal seven: School violence content area. En R. Talley y G. Walz (Eds.), Safe schools, safe students (pp. 85-102). Wasington DC: The National Education Goals Panel and theNational Alliance of Pupil Services Organizations.

Furlong, M. y Morrison, G. (2000). The school in school violence: Definitions and facts. Journal of Emotional and Behavioral Disorders, 8, 71-81.

Hinduja, S. y Patchin, J.W. (2007). Cyberbullying victim and offender warning sings. Recuperado el 3 de noviembre de 2007 de http://www.cyberbullying.us/cyberbullying_warning_sings.pdf

Hyman, I. y Perone, D. (1998). The other side of school violence: Educator policies and practices that may contribute to student misbehavior. Journal of School Psychology, 36 (1), 7-27.

Lacasse, A., Purdy, K.T. y Mendelson, M.J. (2003). The mixed company they keep: Potentially offensive sexual behaviours among adolescents. International Journal of Behavioral Development, 27, 532-540.

Marín, M. (2002). Aspectos psicosociales de la violencia en el contexto educativo. Aula Abierta, 79, 85-105.

Monks, C., Smith, P., Naylor, P., Barter, C., Ireland, J. y Coyne, I. (2009). Bullying in different contexts: Commonalities, differences and the role of theory. Aggression and Violent Behavior, 14, 146-156.
Muñoz, M.C., Pérez, D. y Martín, E. (2006). ¿Qué penalizan los docentes?: análisis de la disciplina a través de los partes de incidencia. Infancia y Aprendizaje, 29, 423-435.

Nansel, T., Overpeck, M., Pilla, R., Ruan, W., Simons-Morton, B. y Scheidt, P. (2001). Bullying behaviors among US youth: Prevalence and association with psychological adjustment. JAMA, 285, 2094-2100.

Nickerson, A. y Martens, M. (2008). School Violence: Associations with Control, Security/Enforcement, Educational/ Therapeutic Approaches, and Demographic Factors. School Psychology Review, 37, 228-243.

Olweus, D. (1991). Bully/victim problems among schoolchildren: Basic facts and effects of a school based intervention program. En D.J. Pepler y K.H. Rubin (Eds.), The development and treatment of childhood aggression (pp. 411-448). Hillsdale, NJ: Erlbaum.

Olweus, D. (1993). Bullying at school: What we know and what we can do. Oxford: Blackwell.

Olweus, D. (1994). Bullying at school: Basic facts and effects of a school based intervention program. Journal of Child Psychology and Psychiatry, 35, 1171-1190.

Owens, L., Daly, A. y Slee, P. (2005). Sex and age differences in victimization and conflict resolution among adolescents in a south Australian school. Aggressive Behavior, 31, 1-12.

Pagani, L., Tremblay, R.E., Nagin, D., Zoccolillo, M., Vitaro, F. y McDuff, P. (2009). Risk factor models for adolescent verbal and physical aggression toward fathers. Journal of Family Violence, 24, 173-182.

Patchin, J.W. y Hinduja, S. (2006). Bullies moves beyond the schoolyard: A preliminary look at cyberbullying. Youth Violence and Juvenile Justice, 4(2), 148-169.

Rigby, K. (2002). New perspectives on bullying. London: Jessica Kingsley.

Rodríguez, F.J., Gutiérrez, C., Herrero, F.J., Cuesta, M. Hernández, E., Carbonero, P.G. y Jiménez, A. (2002). Violencia en el marco escolar de la enseñanza obligatoria: el profesorado como referencia. Aula Abierta, 79, 139-152.

Salmivalli, C., Lagerspetz, K., Björkqvist, K., Österman, K. y Kaukiainen, A. (1996). Bullying as a group process: participant roles and their relations to social status within the group. Aggressive Behavior, 22, 1-15.

Sawyer, A.L., Bradshaw, C. y O’Brennan, L. (2008). Examining Ethnic, Gender, and Developmental Differences in the Way Children Report Being a Victim of "Bullying" on Self-Report Measures. Journal of Adolescent Health, 43, 106-114.

Scheithauer, H., Hayer, T., Peterman, F. y Jugert, G. (2006). Physical, verbal, and relational forms of bullying among German students: Age trends, gender differences, and correlates. Aggressive Behavior, 32, 261-275.

Smith, P. K., Mahdavi, J., Carvalho, M., Fisher, S., Russell, S. y Tippett, N. (2008). Cyberbullying: Its nature and impact in secondary school pupils. Journal of Child Psychology and Psychiatry, 49, 376-385. 


\section{UN ESTUDIO DESCRIPTIVO SOBRE EL ACOSO Y VIOLENCIA ESCOLAR EN LA EDUCACIÓN OBLIGATORIA}

Smith, P. K., Morita, Y., Junger-Tas, J., Olweus, D., Catalano, R. y Slee, P. (1999). The nature of school bullying: A crossnational perspective. London \& New York: Routledge.

Sourander, A. y Helstelä, L. (2000). Persistence of bullying from childhood to adolescence -A longitudinal 8-year follow-up study. Child Abuse and Neglect, 24, 873-881.

Wolke, D., Woods, S., Bloomfield, L. y Karstadt, L. (2000). The association between direct and relational bullying and behaviour problems among primary school children. Journal of Child Psychology and Psychiatry, 41, 989-1002.
Yoon, J.S. (2004). Predicting teacher interventions in bullying situations. Education and Treatment of Children, 27, 37-45.

Fecha de recepción: 16 de abril de 2009

Fecha de aceptación: 24 a abril de 2009 